\title{
Adaptive Selection Combiner over Log-normal Fading Channel in Wireless Communication System
}

\author{
Adeyemo Z. K. ${ }^{1}$, Ojo S. I. ${ }^{1, *}$, Omowaiye R. O. ${ }^{1}$, Bamikefa I. A. ${ }^{2}$ \\ ${ }^{1}$ Department of Electronic and Electrical Engineering, Ladoke Akintola University of Technology, Nigeria \\ ${ }^{2}$ Department of Electronic and Electrical Engineering, Federal Polytechnic, Nigeria
}

Received June 3, 2020; Revised July 13, 2020; Accepted August 10, 2020

\section{Cite This Paper in the following Citation Styles}

(a): [1] Adeyemo Z. K., Ojo S. I., Omowaiye R. O., Bamikefa I. A. , "Adaptive Selection Combiner over Log-normal Fading Channel in Wireless Communication System," Universal Journal of Electrical and Electronic Engineering, Vol. 7, No. 5, pp. 263 - 272, 2020. DOI: 10.13189/ujeee.2020.070501.

(b): Adeyemo Z. K., Ojo S. I., Omowaiye R. O., Bamikefa I. A. (2020). Adaptive Selection Combiner over Log-normal Fading Channel in Wireless Communication System. Universal Journal of Electrical and Electronic Engineering, 7(5), 263 - 272. DOI: 10.13189/ujeee.2020.070501.

Copyright@2020 by authors, all rights reserved. Authors agree that this article remains permanently open access under the terms of the Creative Commons Attribution License 4.0 International License

\begin{abstract}
Wireless communication system has found worldwide acceptability in providing acceptable services due to its portability, flexibility and ease of usage. However, the system is characterized by severe multipath propagation effects that degrade its performance. Selection Combiner (SC) as one of the techniques being used to address this problem is associated with poor performance due to fixed constellation of the modulation scheme. Hence, in this paper, an Adaptive Selection Combiner (ASC) using Constellation Adaptation Algorithm (CAA) with an expression over Log-normal fading channel is proposed. ASC is developed using conventional SC (CSC) and CAA. Ten thousand randomly generated bits are modulated using each of Phase Shift Keying (PSK) and Quadrature Amplitude Modulation (QAM) schemes. The modulated signals at varying paths are scanned by CSC to choose a path with the highest Signal to Noise Ratio (SNR). The output of SC which is a function of Channel State Information (CSI) acquired is used to determine the channel gain. The constellation size is then adjusted based on the value of channel gain through CAA. The proposed adaptive technique is simulated using MATLAB software and evaluated using Bit Error Rate (BER) to determine its performance. The results obtained show that ASC gives lower BER values than CSC due to the self-adjustment of the constellation size. Therefore, the proposed ASC can be deployed to improve the performance of wireless communication systems.
\end{abstract}

Keywords Constellation Adaptation Algorithm (CAA),
Selection Combining (SC), Adaptive Modulation, Channel State Information (CSI), Bit Error Rate (BER), Log-Normal Fading Channel and Channel Gain

\section{Introduction}

Wireless Communication (WC) which is the act of conveying information from one point to another through an unbounded environment has been receiving much attention in the field of technology due to distance limitation in a wired medium [1, 2]. It is governed by the transmission medium which is dynamic and unpredictable. The dynamic and unpredictable nature of the medium makes an exact analysis of radio wave propagation often difficult $[1,3]$. In WC, radio waves are mainly affected by some propagation mechanisms such as reflection, diffraction, refraction and scattering of signals leading to multipath propagation. Multipath propagation is a phenomenon that occurs when a transmitted signal propagates through many paths as a result of obstructions in the environment. The resulting effect of the multipath propagation is the variability of the received signal due to different paths having different angles of arrival and time $[2,4]$. In order to design an effective wireless communication system that has a good performance to meet-up with an exponential increase in its services, the characteristics of the radio channel in which systems 
operate need to be considered. The performance of the system depends on some signal processing techniques at the transmitter and receiver such as amplification, coding, modulation, filtering as well as the nature of the channel at a particular time [5, 6].

Modulation which is one of the signal processing techniques used at the transmitter to modify the signal parameters into a form suitable for transmission over propagating medium [1]. When the signal parameters vary in accordance with the binary digit known as modulating signal result in Digital Modulation (DM). DM is widely used in WC due to its greater noise immunity and robustness to channel impairments. DM schemes include Phase Shift Keying (PSK), Quadrature Amplitude Modulation (QAM) and Frequency Shift Keying (FSK). PSK and QAM are used in this paper due to their robustness to channel impairments during signal propagation. PSK involves varying the phase of baseband signal in accordance with the phase of the carrier signal, while QAM modifies the phase and amplitude of the signal simultaneously in accordance with carrier signal $[1,7,23]$. The number of bits transmitted per symbol in wireless communication is a function of modulation order known as a constellation. Higher-order modulation permits many bits per symbol and easily distorted if the channel is in the worst situation. However, lower constellation allows few bits per symbol to be transmitted and robust in the channel $[4,8]$. Wireless systems are equipped with channel measurement to know the status of the channel at a particular time. This allows the receiver to measure the fade depth of the received signal which may be controlled by adjusting the schemes constellation for suitable transmission. Such control is achieved in various ways such as adjusting the power level, symbol rate, coding scheme, constellation size, and any combination of these approaches. The receiver detects these changes directly from the received data without the need for an explicit rate change control information. This paper focuses on adjusting the constellation size of modulation which is referred to adaptive modulation [9, 10]. Adaptive Modulation (AM) scheme adjusts the constellation with respect to time-varying channels to have better performance. The essence of AM is to feedback the Channel State Information (CSI) from the receiver to transmitter, so that the constellation in the transmitter can adjust the constellation based on information with respect to the channel conditions. It allows users to send more bits per symbol and thus achieve higher throughputs [9]. AM has the potential to increase the system throughput significantly by matching transmitter parameters to time-varying channel conditions, thereby combating the effects of multipath fading in a wireless environment [11].

Some fading compensation diversity combining techniques have been in existence to mitigate the effects of fading. These are Equal Gain Combiner (EGC), Maximal Ratio Combiner (MRC), Selection Combiner (SC) and Threshold Combiner (TC). The result of past researches on diversity combining technique revealed that SC and TC are often used in practice because of their reduced complexity but have poor performances compared to MRC and EGC. The poor performance is due to using only one path out of the $L$ available paths, fixed modulation constellation, and time-varying nature of the channel [3]. Therefore, this paper proposes an adaptive SC that adjusts the constellation based on the Channel State Information (CSI) through Constellation Adaptation Algorithm (CAA). CAA allows the transmitter to adapt the constellation size of the modulation scheme to the radio channel conditions. In CAA, the transmitter chooses from a finite set of constellations with the aim of identifying the best constellation suitable to provide maximum throughput at the higher data rate. It involves the use of optimal constellation for a given channel quality and the poorer the channel quality, the lower the constellation should be and vice versa. The statistical distribution of multipath propagation in time-varying channel follows different fading distributions such as Nakagami-m distributions, Rayleigh distribution and Log-Normal distribution. In this paper, Log-Normal distribution is adopted due to its application in modeling both the indoor and outdoor environments [3, 4]. The Log-Normal distribution is used in modelling cellular wireless networks in which vegetation and foliage are significant factors that cause scattering and absorption of radio waves making the power of received signal varies at the receiver due to shadowing. It involves fading over large distance causes random fluctuations in the mean signal power while other fading channel such as Rayleigh, Rician and Nakagami-m are fading over small distance and time [4].

\section{Related Works}

In [12] analysis of the performance of Selection Combining (SC) over-generalized Rayleigh channel in solving signal fluctuation at the receiver due to multipath fading was carried out. The instantaneous SNR of all the received paths were compared with one another to select the path with the highest value of SNR. The selected path was used as the output and there was no signal co-phasing since only one path was used at a time. The results obtained revealed that SC had a poor performance with higher Bit Error Rate (BER) than other combining techniques due to fixed constellation used in the system. BER analysis of EGC with co-channel interference was presented in [13] to mitigate signal fluctuation at the receiver due to multipath fading. The multiple copies of the received signals were weighted with equal weight and co-phased to avoid signal cancellation. The output signals were multiplied with equal weight and summed up to obtain the output signals. The results obtained showed that the technique had better performance with lower BER but suffered from hardware complexity. Also, [14] worked on Maximal Ratio Combiner (MRC) with antenna diversity to address the variability of the signal at the receiver due to multipath 
fading. The multiple copies of the received signals were multiplied with different weights and co-phased before summing up to obtain the output signal. Mathematical expression for BER was derived and used to measure the performance of the MRC technique. Results obtained revealed that, MRC had the best performance but suffers from the highest hardware complexity. Furthermore, hybrid diversity combiner over Nakagami fading channel was presented in [2] to address the problem of the hardware complexity of conventional EGC using hybrid Threshold-Equal Gain Combiner (TC-EGC). The multiple copies of the signal were received by multiple TC to select the strongest path and avoid the weak ones. The outputs signal of TC were used as input to EGC and weighed equally before summing. The results obtained showed that, hybrid TC-EGC had better performance with lower BER than other diversity combiner but at the expense of hardware complexity when compared with SC. However, previous researches showed that MRC, EGC and hybrid diversity combining techniques have better performances only in time-invariant channels compared to Selection Combining but at the expense of hardware complexity. Selection Combining (SC) technique being used in practice due to its lower complexity has poor performance compared to MRC and EGC in time-varying channel. The poor performance is due to fixed constellation used in the technique. Therefore, this paper focuses on the self-adjustment of constellation size based on the Channel State Information (CSI) to improve the performance of SC technique.

\subsection{Conventional Selection Combiner}

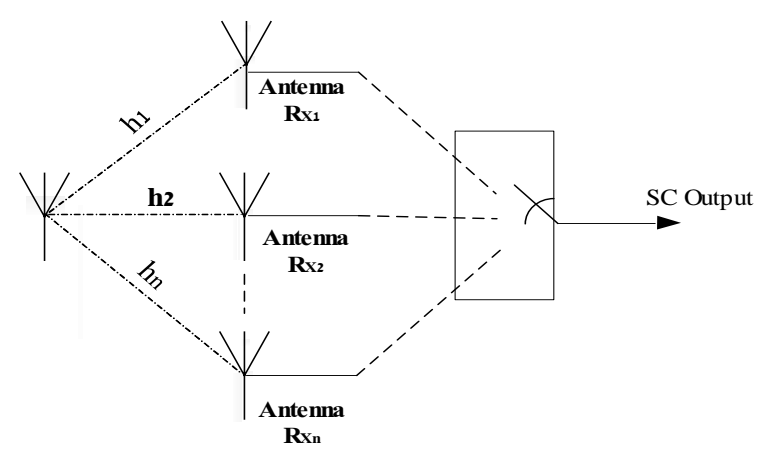

Figure 1. Block diagram of CSC [17]

In Conventional Selection Combiner (CSC), the combiner gives the signal on the branch with the highest SNR. The output of the SC combiner is equal to the signal on only one of the branches, the coherent sum of the individual branch signals is not required. Since only one branch is used at a time, SC often requires just one receiver that is switched into the branch with the highest SNR [15]. Moreover, since only one branch output is used, the co-phasing of multiple branches is not required, unlike MRC and EGC that require all the CSI from all the received signals $[15,16]$. Therefore, the SC technique is used in conjunction with differentially coherent and non-coherent modulation techniques since it does not require knowledge of the signal phases on each branch like MRC or EGC in a coherent system. The general form of conventional SC is to monitor all the diversity branches and select the best one for detection [15]. As a result of this, it is right to say that, SC is not a combining method but a selection process at the available branches [16, 25]. Fig. 1 shows the block diagram of SC in which the branch with the highest signal strength is selected at a particular time. CSC suffers from poor performance due to only one branch and fixed constellation used.

\subsection{Adaptive Modulation}

Adaptive Modulation (AM) is a technique that maximizes the data throughput of signals allocated to a user. It involves measuring the SNR of each path in the transmission, then, selecting a modulation scheme that maximizes the spectral efficiency to maintain an acceptable performance [22, 24]. AM is one of the techniques used to automatically select the different constellations in accordance with the severity of the fading channel. The response to channel variation is according to channel adaptation parameters such as constellation change, coding, and other characteristics of the signal for better reception $[18,19]$. AM technique is based on the idea that when a certain branch of the signal is corrupted by fading, smaller dimension constellation and more transmission power are assigned to this particular branch. Higher-order constellations and less transmission power are assigned to the branch signal whose channel gain is high. To achieve the data rate of 2 bps per branch of signal, BPSK modulation was used over the faded channel and the 8PSK modulation at the non-faded branch of the signal [18, 20, 26].

\section{Proposed Adaptive Selection Combiner}

The randomly generated signal is propagated through a Log-normal fading channel from the transmitter which consists of each of the M-ary PSK and QAM signal processing techniques for obtaining different constellations. The conventional SC scans through the multiple copies of the signals to choose the one with the highest SNR $(\max R(t))$ as shown in Fig. 2. The selected signal passes through CSI to obtain the channel gain. The output of CSI is used as input to the decision device to determine the channel condition based on the value of channel gain obtained. The transmitter then selects the appropriate constellation size for another transmission of the slot through the CAA. The flowchart of the MATLAB used for simulation of the proposed ASC technique is shown in Fig. 3. 


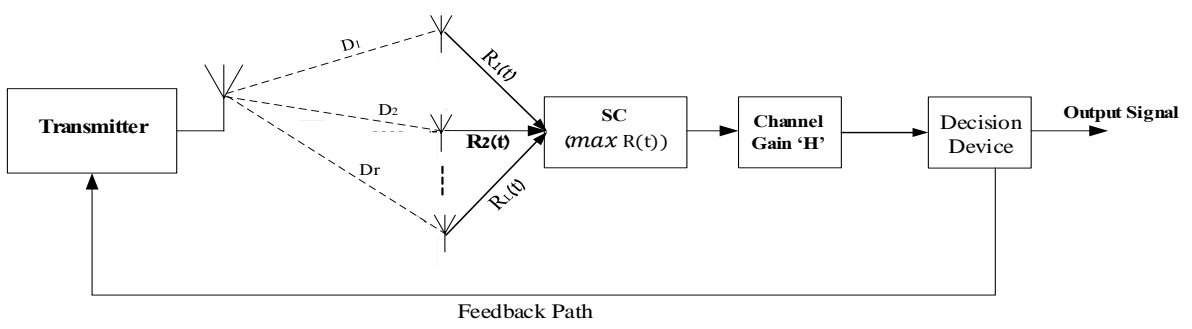

Figure 2. Block diagram of an adaptive SC

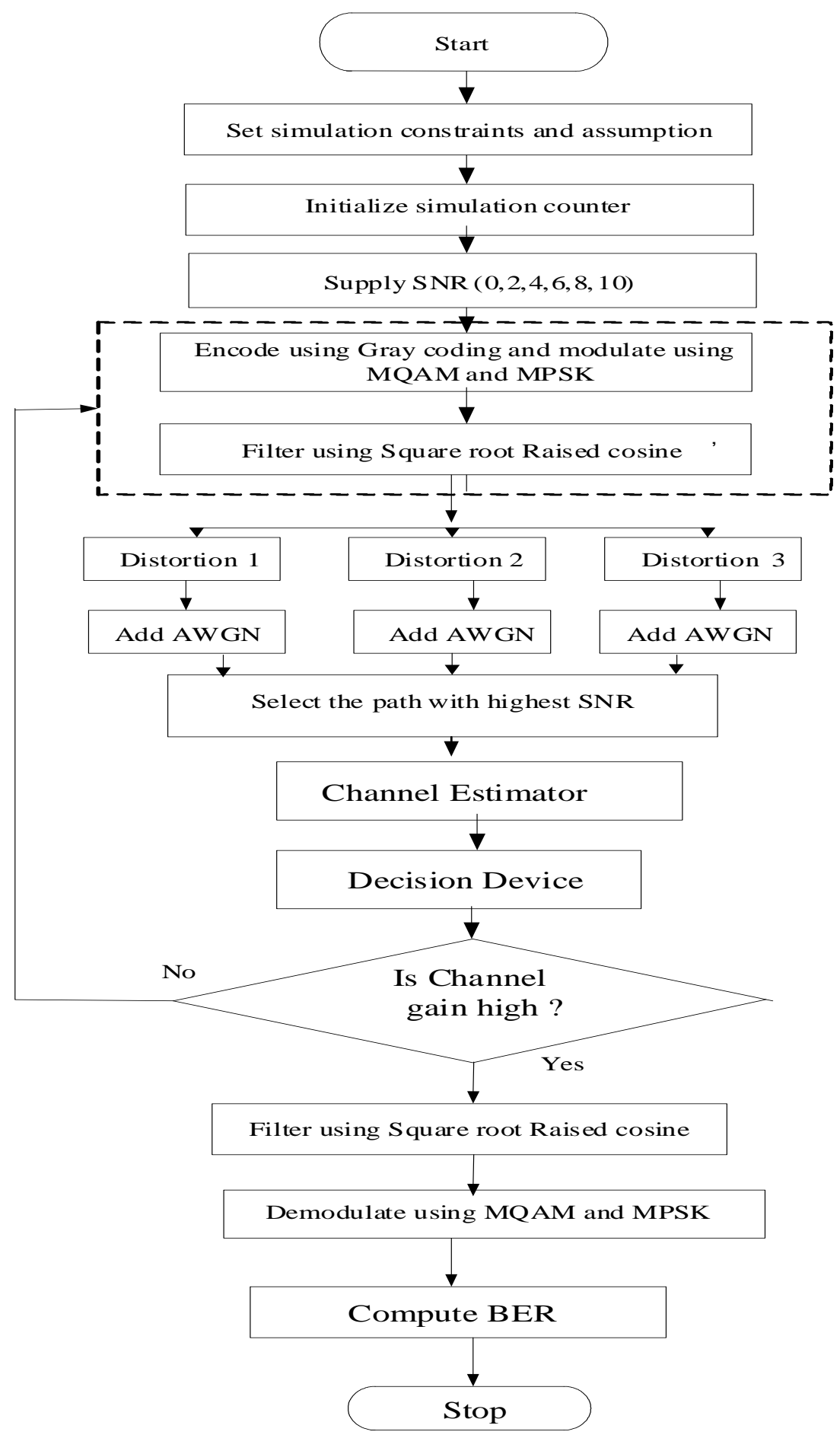

Figure 3. Flowchart of the MATLAB simulation process for the proposed ASC technique 


\subsection{Probability Density Function (PDF) of the Received Signal for the Proposed Technique}

The instantaneous SNR of the received signal ' $\gamma$ ' is given by [10] as the product of channel gain ' $H^{\prime}$ at a particular time and the constant $P_{t} / N B$. That is,

$$
\gamma=\frac{P_{t} H}{N B}
$$

where: $P_{t}$ is the transmit power

$B$ is the channel bandwidth

$H$ is the channel gain

$N$ is the noise spectral density

But PDF of the signal propagated over an uncorrelated Log-normal fading channel ' $P_{r}(\gamma)$ ' is expressed by [10] as

$$
P_{r}(\gamma)=\frac{4.3429}{(2 \pi \gamma \sigma)^{\frac{1}{2}}} \exp \left(-\frac{\left(10 \log _{10} \gamma(L)-\mu\right)}{2 \sigma^{2}}\right)^{2}
$$

where: $\gamma$ is the received signal

$\mathrm{L}$ is the number of paths

$\mu$ and $\sigma$ are the mean and the standard deviation of the energy of the received signal, respectively

But, the Bit Rate ' $\mathrm{R}$ ' is given by [21] as

$$
R=B \times \log _{10}\left(\frac{1+(P \times H)}{N}\right)
$$

From equation (3), channel gain ' $H$ ' is derived as follows

$$
\begin{gathered}
\frac{R}{B}=\log _{10}\left(\frac{1+(P \times H)}{N}\right) \\
10^{\frac{R}{B}}=\frac{1+(P \times H)}{N} \\
H=\frac{\left(N \times 10^{R / B}\right)-1}{P}
\end{gathered}
$$

Substituting equation (6) into equation (1) gives

$$
\gamma=\frac{P_{t} \frac{\left(N \times 10^{R / B}\right)-1}{P}}{N B}
$$

Since $P_{t}=P$, therefore,

$$
\gamma=\frac{\left(N \times 10^{R / B}\right)-1}{N B}
$$

Equation (8) is the instantaneous SNR of the received signal for the proposed technique. Therefore, substituting equation (8) into (2) yields

$$
\begin{gathered}
P_{r}(\gamma) \frac{4.3429}{(2 \pi \gamma \sigma)^{\frac{1}{2}}} \exp \left(-\frac{\left(10 \log _{10}\left(\left(N \times 10^{R / B}\right)-1\right)-\mu\right)}{N B 2 \sigma^{2}}\right)^{2} \\
P_{r}(\gamma) \frac{4.3429}{(2 \pi \gamma \sigma)^{\frac{1}{2}}} \exp \left(\frac{-5 \log _{10}\left(\left(N \times 10^{R / B}\right)-1\right)-\mu}{N B \sigma^{2}}\right)^{2}
\end{gathered}
$$

\subsection{Bit Error Rate for the Proposed Technique}

The performance of the proposed technique is evaluated using Bit Error Rate (BER). According to [7], the expression for BER is given as

$$
B E R=\int_{o}^{\infty} P_{b}(E / \gamma) P_{r}(\gamma) d \gamma
$$

where: $P_{b}(E / \gamma)$ is the conditional error probability

$E$ is signal energy per symbol

For non-coherent modulation, conditional error probability $P_{b}(E / \gamma)$ is given by [27] as

$$
P_{b}(E / \gamma)=1 / 2 \exp (0.5 \gamma)
$$

Substituting equation (12) into (11) gives

$$
B E R=\int_{o}^{\infty} 1 / 2 \exp (0.5 \gamma) P_{r}(\gamma) d \gamma
$$

The PDF of the output SNR of the received signal is given in equation (10). Therefore, substituting equation (10) into (13) yields

$$
\begin{array}{r}
B E R= \\
\int_{0}^{\infty} 1 / 2 \exp (0.5 \gamma) \frac{4.3429}{(2 \pi \gamma \sigma)^{\frac{1}{2}}} \exp \left(\frac{-5 \log _{10}\left(\left(N \times 10^{R / B}\right)-1\right)-\mu}{N B \sigma^{2}}\right)^{2} d \gamma
\end{array}
$$

$$
B E R=\frac{4.3429}{(4 \pi \gamma \sigma)^{\frac{1}{2}}} \int_{o}^{\infty} \exp \left((0.5 \gamma)-\frac{5 \log _{10}\left(\left(N \times 10^{R / B}\right)+\mu\right)}{N B \sigma^{2}}\right)^{2} d \gamma
$$

Algorithm 1: Constellation Adaptation Algorithm

1: Begin

2: Initialize R, B, N, P

3: $\mathrm{C}_{\mathrm{i}}=128 \mathrm{QAM}, 128 \mathrm{PSK}$

4: compute SNR of the received signals

5: select max SNR

6: compute $\mathrm{H}$ for the selected branch using (6)

7: if $(\mathrm{H}>=0.1)$ or $(\mathrm{H}<=0.25)$ then

8: new constellation size (C1) $=4 \mathrm{QAM}$, $4 \mathrm{PSK}$

9: elseif $(\mathrm{H}>=0.26)$ or $(\mathrm{H}<=0.45)$ then

10: new constellation size $(\mathrm{C} 2)=8 \mathrm{QAM}$, 8PSK

11: elseif $(H>=0.46)$ or $(H<=0.65)$ then

12: new constellation size $(\mathrm{C} 3)=16 \mathrm{QAM}, 16 \mathrm{PSK}$

13: elseif $(H>=0.66)$ or $(H<=0.85)$ then

14: new constellation size (C4) $=32 \mathrm{QAM}, 32 \mathrm{PSK}$

15: elseif $(\mathrm{H}>0.85)$ then

16: new constellation size (C5) = 64QAM, 62PSK

17: else

18: new constellation size $=\mathrm{C}_{\mathrm{i}}$

19: End

\section{Simulation Results and Discussion}

Figs. 4, 5 and 6 present the Bit Error Rate (BER) against SNR for Adaptive Selection Combiner (ASC) and Conventional Selection Combiner (CSC) at different paths $(\mathrm{L}=2,3,4)$ using PSK signaling scheme over the 
Log-normal fading channel. Fig. 4 depicts BER against SNR for ASC and CSC at L of 2 with PSK scheme. At SNR of $6 \mathrm{~dB}$, the BER values of 0.2738 and 0.4484 are obtained for ASC and CSC, respectively, while the corresponding BER values of 0.1055 and 0.3376 are obtained for ASC and CSC, respectively with $L$ of 3 as depicted in Fig. 5. Fig. 6 depicts the BER values obtained using PSK modulation scheme and $L$ of 4 . The BER values obtained at SNR of 6 $\mathrm{dB}$ are 0.0227 and 0.2936 for ASC and CSC, respectively. The results obtained show that BER values decrease with increase in the number of paths due to reduction in error rate as signal strength increases. Figs. 7, 8 and 9 show the BER results obtained using QAM modulation schemes at $L=2,3$ and 4 , respectively. Fig. 7 presents the BER results with L of 2 using QAM signaling scheme over the Log-normal fading channel for ASC and CSC. At SNR of 6 $\mathrm{dB}$, the BER values obtained are 0.2791 and 0.4091 for ASC and CSC, respectively, while 0.1004 and 0.3512 are the corresponding values for ASC and CSC, respectively with $\mathrm{L}$ of 3 at SNR of $6 \mathrm{~dB}$ as presented in Fig. 8. Fig. 9 depicts the BER values at $\mathrm{L}$ of 4 using the QAM scheme, at SNR of $6 \mathrm{~dB}$, BER values of 0.0341 and 0.3062 are obtained for ASC and CSC, respectively. Results obtained show that ASC gives lower BER values than CSC and this is due to self - adjustment of the proposed technique. Also, BER decreases as the number of path and SNR increase. This is due to the fact that, the signal strength increases as the number of path as well as SNR increases.

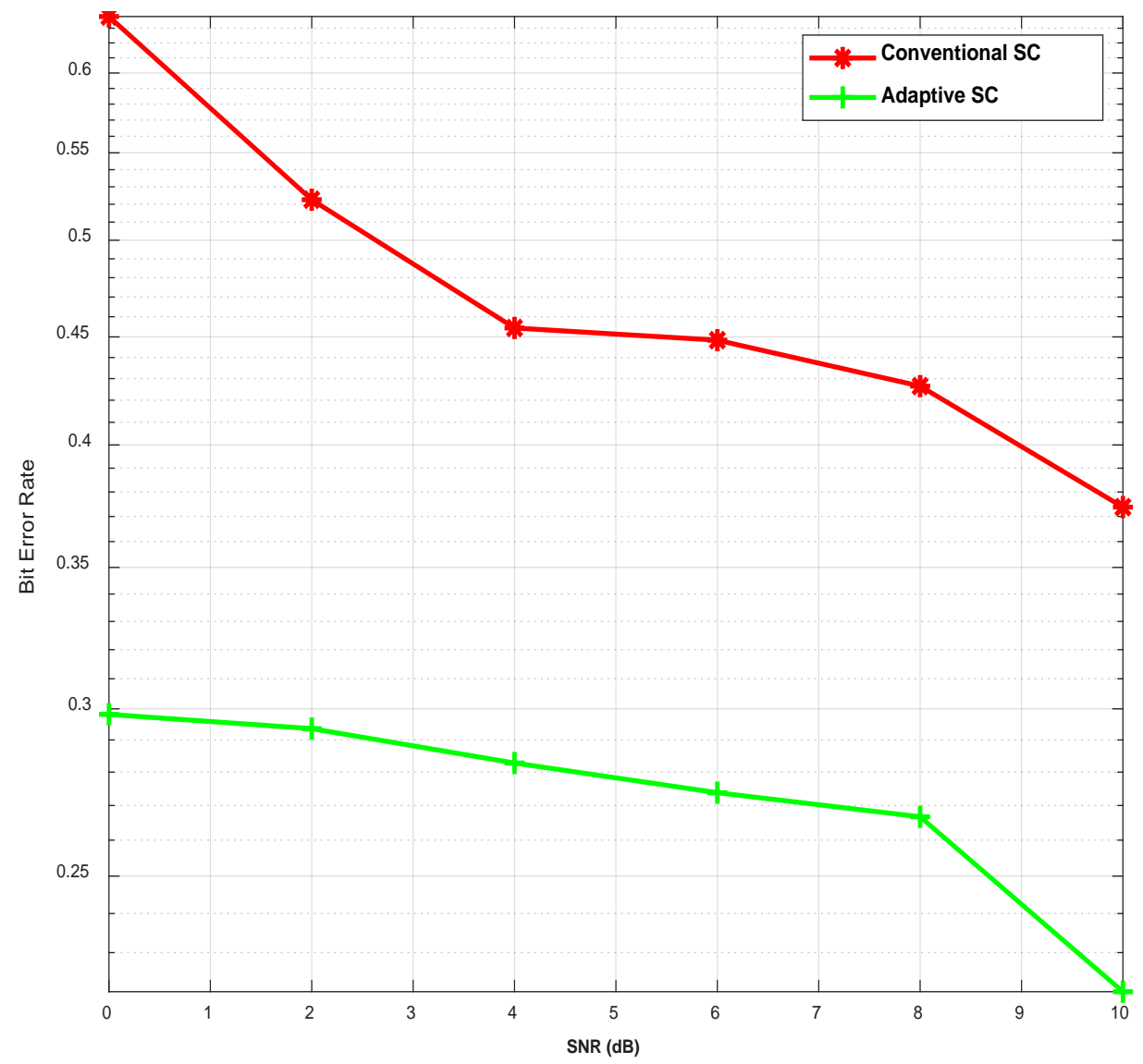

Figure 4. Bit Error Rate against SNR for ASC and CSC at $L=2$ with the PSK modulation scheme over Log-normal Fading Channel. 


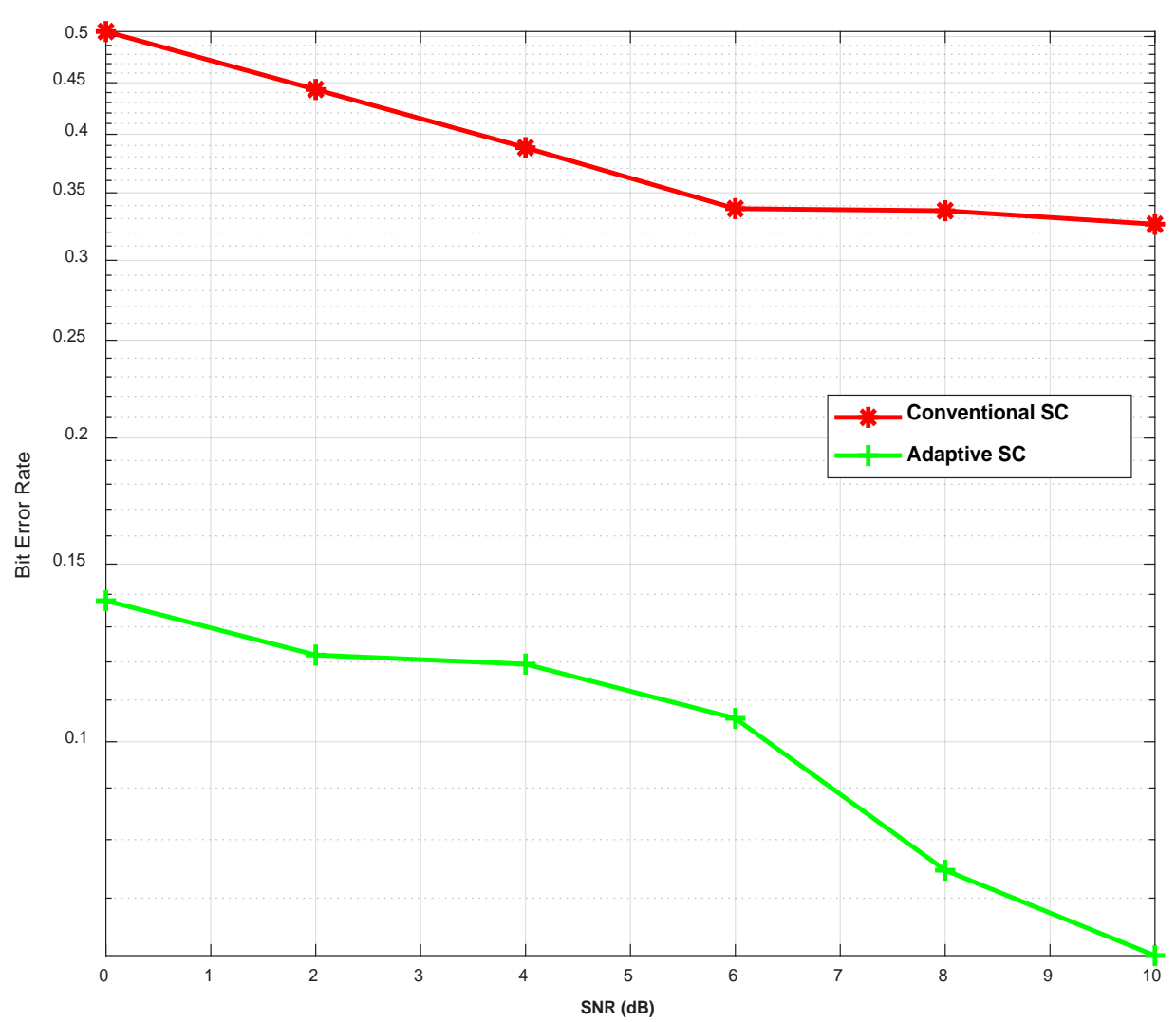

Figure 5. Bit Error Rate against SNR for ASC and CSC at $L=3$ with PSK modulation scheme over Log-normal Fading Channel

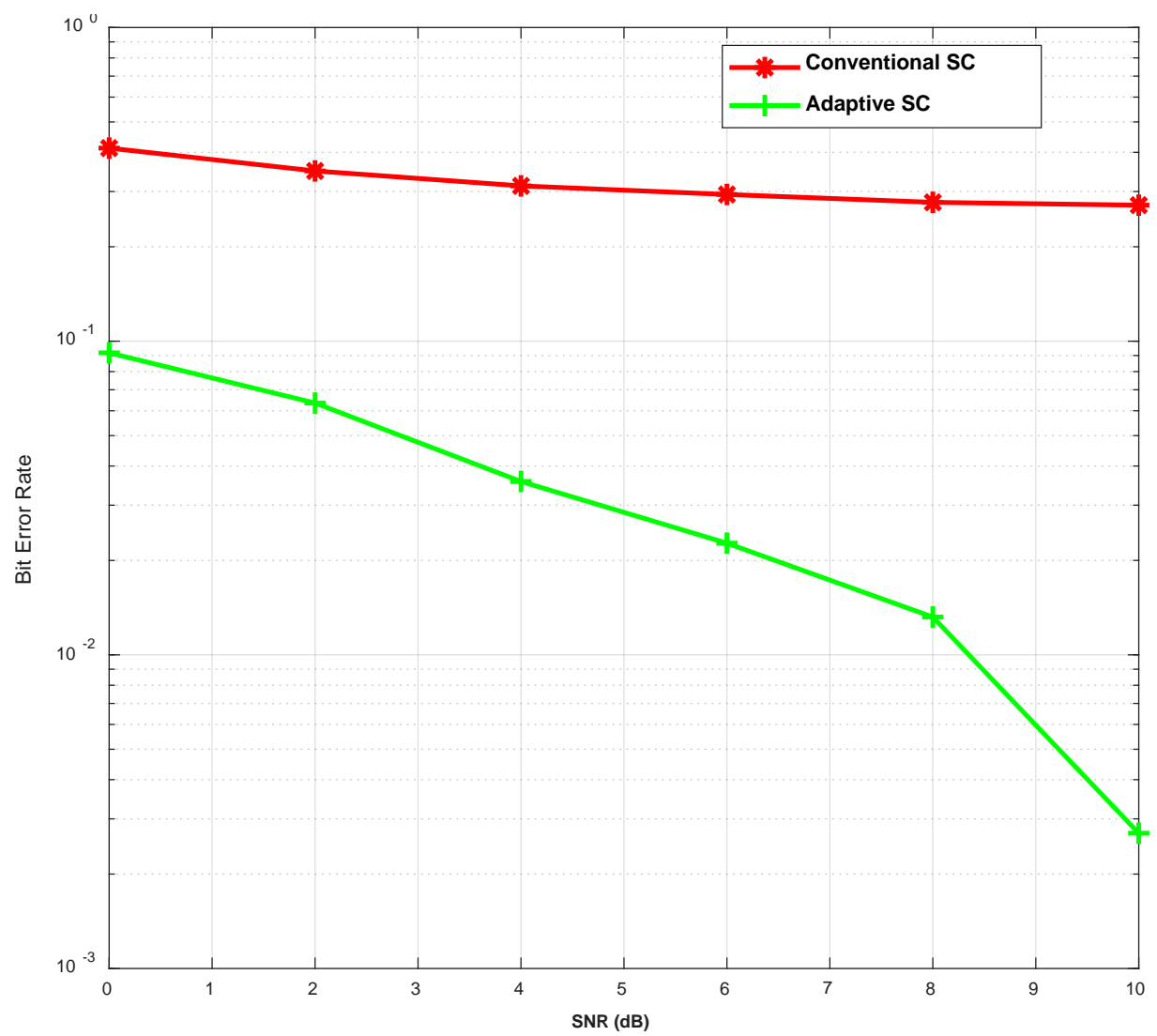

Figure 6. Bit Error Rate against SNR for ASC and CSC at $L=4$ with the PSK modulation scheme over Log-normal Fading Channel 


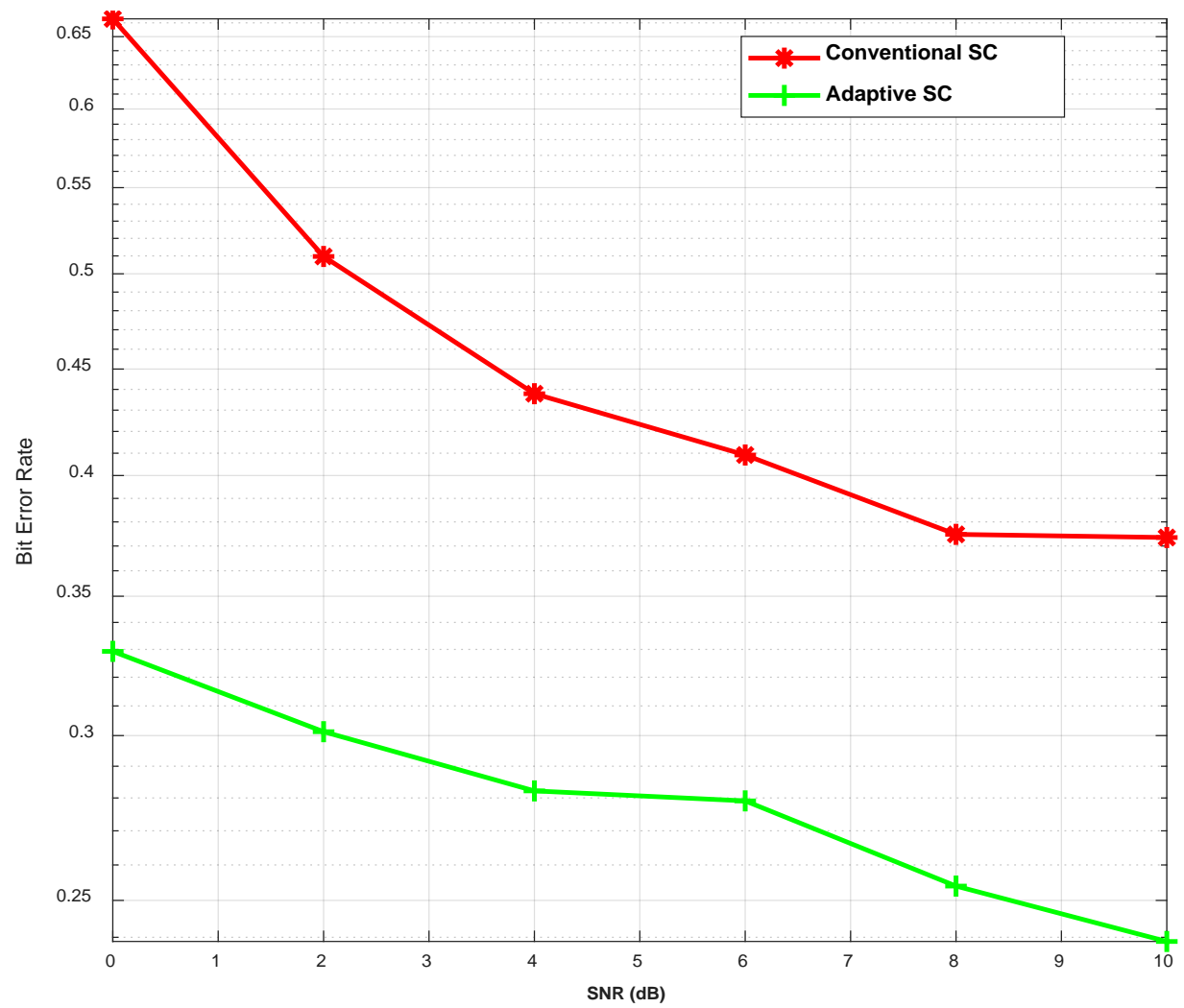

Figure 7. Bit Error Rate against SNR for ASC and CSC at $L=2$ using QAM modulation scheme over Log-normal Fading Channel

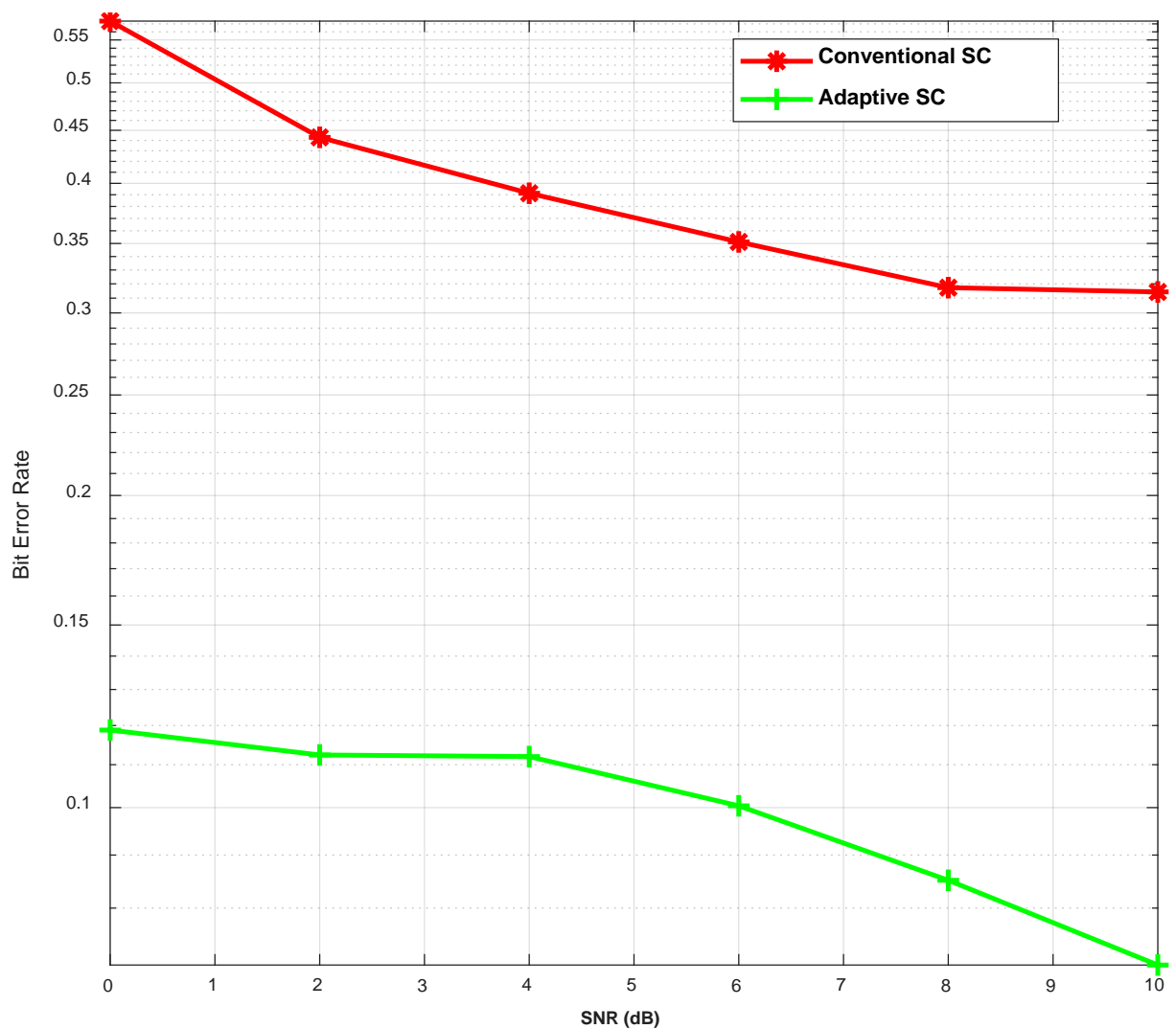

Figure 8. Bit Error Rate against SNR for ASC and CSC at $L=3$ with QAM modulation scheme over Log-normal Fading Channel 


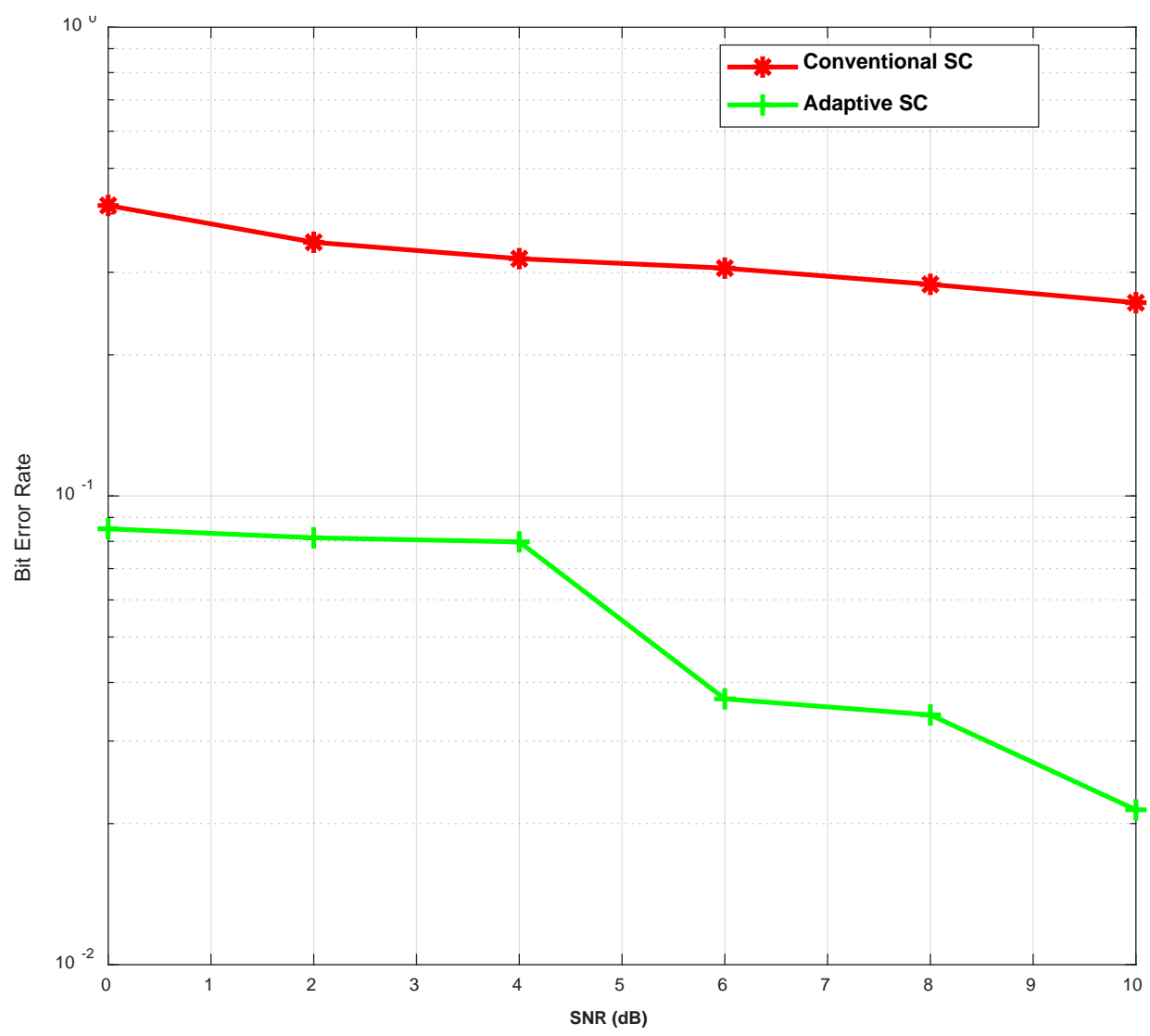

Figure 9. Bit Error Rate against SNR for ASC and CSC at $L=4$ using QAM modulation scheme over Log-normal Fading Channel

From the results obtained, ASC shows a better performance with lower values of BER than the conventional SC at $L=2,3$, and 4 using PSK and QAM modulation schemes. The better performance of the proposed adaptive SC scheme is due to self-adjustment which is achieved by obtaining channel gain at a particular time using CSI and instructs the transmitter incorporating the modulation to increase or decrease the constellation size based on the value of channel gain via CAA. The results obtained also reveal that, BER decreases as the number of paths increases.

\section{Conclusions}

In this paper, an Adaptive Selection Combining (ASC) technique has been proposed using Channel State Information (CSI) over the Log-normal fading channel. Closed-form expression for Probability Density Function (PDF) of the received signal is derived for the proposed adaptive technique and used to derive Bit Error Rate (BER) expression to evaluate the performance of the proposed technique. BER values for adaptive and conventional SC are obtained at different SNRs using different paths and modulation schemes. Performance of the ASC and CSC have been evaluated at different paths ' $\mathrm{L}$ ' with different constellations for PSK and QAM modulation schemes using Bit Error Rate as a performance metric. The results obtained reveal that ASC performs better than CSC due to lower BER values obtained. The better performance of ASC is a result of the selection of constellations size based on CSI which makes the technique to be self-adjusted. Therefore, adaptive SC has been shown to have a better performance over CSC.

\section{REFERENCES}

[1] Zachaeus K. Adeyemo, Isaac A. Ojedokun and Damilare O. Akande." Symbol Error Rate Analysis of M-QAM with Equal Gain Combining over a Mobile Satellite Channel” International Journal of Electrical and Computer Engineering, 3(6):849-856, 2013

[2] Zachaeus K. Adeyemo, Samson I. Ojo, Simeon B. Ebinaiye and Olasunkanmi F. Oseni. Development of a Hybridized Diversity Combiner over Nakagami Fading Channel, International Journal of Information Engineering and Electronic Business, 6(3):45-53, 2019.

[3] Adeyemo, Z.K, and Ojedokun, I.A. EGC Receiver using Single Radio Frequency Chain and Single Matched Filter over Combined Rayleigh and Rician Fading Channels; ARPN Journal of Engineering and Applied Sciences, 9(7) 992-994, 2014

[4] Ojo, S.I, Abolade, R.O, Adeyemo, Z.K., Akintoye, N.O and 
Salami, H.T. Enhancement of Maximal Ratio Combiner over Log-Normal Fading Channel using Firefly Algorithm, Transactions on Networks and Communications, 7(2): 1-11, 2019.

[5] Adeyemo, Z.K., Badrudeen and Abolade, R.O. Intersymbol Interference Distortion Cancellation Using a Modified Maximal Ratio Combiner in Mobile Wireless Communication; Journal of Information Engineering and Application, 3(8): 16-17, 2013.

[6] Rajkumar G. Performance Evaluation of Maximum Ratio Combining Scheme in WCDMA System for different Modulation, International Journal of Emerging Technologies in Engineering Research 4(5):240-242, 2016.

[7] Rappaport, T.S. wireless communications principle and practice, Even publishers Chicago, pp 240-250, 2002.

[8] Huang, J.L. Adaptive MIMO Systems with Channel State Information at Transmitter. Unpublished Ph.D thesis, University of Stockholm, Sweden, pp 67-167, 2009.

[9] Suvarna, P.J and Vaibhav, S.H. Performance of Maximum Ratio Combining (MRC) MIMO Systems for Rayleigh Fading Channel, International Journal of Scientific and Research Publications, 3(2):1-3, 2013.

[10] Simon, M.K. and Alouini, M.S. Digital Communication over fading channels. John Wiley \& Sons. Inc. Hoboken, New Jersey, pp 34-937, 2005.

[11] Mohamed, S. and Marvin, K.S. Performance of Coherent Receiver with Hybrid SC/MRC over Nakagami-m Fading Channel; IEEE Transactions on Vehicular Technology 48(4):1155-1157, 1999.

[12] Vinay, K.P. and Sirisha, M. Performance analysis of selection combining diversity scheme in generalized Rayleigh fading channel, IEEE International conference on communications and signal processing, Melmaru Vathur, pp 3-20, 2014.

[13] Aruna, G. BER of equal gain combiner with co-channel interference, IEEE International conference on computer communication and informatics, India, pp 6-15, 2017.

[14] Stevan, M.B. and Kevin W.S. Maximal Ratio Combining with antenna diversity, International Journal of Wireless Communication and Mobile Computing, 9(2): 1-5, 2017.

[15] Stuber, G.L. Principles of Mobile Communications, Second Edition. Kluwer Academic Publishers New York, pp 45-90, 2002.

[16] Ramanthan, V. Performance Evaluation of Equal Gain
Diversity Systems in Fading Channel. Unpublished Master Thesis the Virgiria Polytechnic, Institute and State University pp.69-120, 2003

[17] Ibrahim A. Performance of M-QAM over Generalized Mobile Fading Channel Using MRC Diversity. Unpublished master thesis, College of Engineering, King Saud University, pp. 6-79, 2007.

[18] Armanious, E., Falconer, D.D. and Yanikomergolu, H. Adaptive Modulation, Coding and Power Control in a broadband fixed cellular Wireless System; Journal of Wireless Communication and networking, 1(7): 238-242, 2003.

[19] Mohammed, I. Adaption in Wireless Communication. Taylor and Francis Group. New York pp. 40-83, 2009.

[20] Pratt, T., Boastian, C. W., and Allnutt J.E. Satellite Communications, Second Edition, John Wiley \& Sons, New Jersey, pp.234-261, 2003.

[21] Annamalai A., and Tellambura C. Performance evaluation of generalized selection Diversity Systems over Nakagami m fading channels, Journal of Wireless Communications and Mobile Computing, 3(2): 99 - 116, 2003.

[22] Shengli Z. and Georgios, B.G. Adaptive Modulation for Multi-Antenna Transmissions with Channel Mean Feedback; IEEE Transactions on Wireless Communications, 5(7): 1-9, 2004.

[23] Sushmaja, K. and Fazal, N. Implementation of Binary Shifting Keying Techniques; International Journal of Engineering Trends and Technology 4(6): 2581- 2582, 2013.

[24] Sung, S.N. Adaptive Power Control for Single and Multiuser Opportunistic System, IEEE Transaction on Wireless Communication, 8(6): 245-267, 2009.

[25] Shukla, C.A., Vaibhav, S.H. and Fazal, N. Performance Evaluation of Maximal Ratio Combining Diversity with Prime Interleaves for Iterative IDMA Receiver, International Journal of Information Engineering and Applications, 1(3): 29-39, 2011.

[26] Verma, P. K., Jain, P., Soni, S. and Raw, R. S. Channel capacity of different adaptive transmission techniques over log-normal shadowed fading environment, $3^{\text {rd }}$ IEEE International Conference on Computing for Sustainable Global Development, pp 3455-3459, 2016.

[27] Hima, P.V. and Seema P. Performance Analysis of Hybrid MRC/EGC Diversity Combining Technique over AWGN Channel; Journal Electronics and Communication Engineering 5(6): 25-28, 2016. 